\title{
Dual RAS blockade—controversy resolved
}

Piero Ruggenenti and Giuseppe Remuzzi

We thank Makani and coworkers for carefully reviewing our News \& Views article (Ruggenenti, P. \& Remuzzi, G. Metaanalyses can misdirect decisions on treatment. Nat. Rev. Nephrol. 9, 311-312; 2013) ${ }^{1}$ that discussed their meta-analysis of the effect of dual versus single-agent reninangiotensin system (RAS) blockade. ${ }^{2}$ Here we summarize our key thoughts on their interesting comments (Makani, H. et al. Dual RAS blockade-unresolved controversy. Nat. Rev. Nephrol. doi:10.1038/ nrneph.2013.82-c1). ${ }^{3}$

It is curious that to test their hypothesis that "...more intense RAS inhibition using more than one RAS blocker versus a single agent would offer more benefit in patients with proteinuric renal disease," Makani et al. compared dual versus single RAS blockade in almost 50,000 patients without proteinuric kidney disease. The few patients with proteinuria who could potentially benefit from dual RAS blockade were considered only in the safety analyses and even treatment effect on proteinuria was overlooked. ${ }^{2}$

The reason why the meta-analysis authors disagree with our statement that aliskiren-based dual RAS blockade combinations are associated with an increased risk of stroke is obscure. The ALTITUDE trial (NCT00549757) was stopped prematurely because of an excess of nonfatal strokes in patients treated with aliskiren, ${ }^{4}$ and the FDA warned that combinations of aliskiren with angiotensin-converting enzyme (ACE) inhibitors or angiotensinreceptor blockers 'are contraindicated in patients with diabetes. ${ }^{5}$

In our News \& Views article, we did not emphasize that dual RAS blockade reduced heart failure hospitalizations by $23 \%$ in the CHARM-Added trial (NCT00634309). In fact, we did not mention this trial (which actually showed a $13 \%$ risk reduction). The data we quoted were from the heart failure trials detailed in Figure 4 of Makani et al.'s meta-analysis. ${ }^{2}$ Of note, these data confirmed that the reduction in risk of heart failure hospitalizations with dual versus single-agent RAS blockade was statistically significant, even when trials in patients without heart failure were considered. ${ }^{2}$ Moreover, the reasons for heart failure hospitalizations include events-such as acute pulmonary oedema and cardiogenic shock - that cannot be disregarded as surrogates. To avoid these life-threatening events, we believe that any patient with heart failure would accept dietary and pharmacological counselling to prevent and manage treatment-induced hyperkalaemia.

Makani et al.'s statement that the inclusion of heterogeneous trials in their meta-analysis "is pertinent to any such analysis and is inevitable" is unacceptable. A priori evaluation of study heterogeneity is mandatory for any rigorous meta-analysis and, in addition to statistical criteria, should take into consideration the various disease mechanisms that may influence treatment effect. ${ }^{6}$ Generalization of data to patient populations that differ from those that drive the results of meta-analyses adversely impacts patient care in everyday clinical practice. ${ }^{6}$ Moreover, the statement that the renoprotective effect of dual RAS blockade "is not supported by controlled data" reveals that Makani and coworkers are not familiar with the concept of controlled studies, which include cohortlongitudinal and case-controlled trials as well as randomized controlled trials. ${ }^{7}$ Data on the renoprotective effect of dual RAS blockade have come from prospective, matched-cohort, long-term controlled trials in patients with chronic nephropathy and residual proteinuria despite ACE inhibitor monotherapy. ${ }^{8,9}$

We disagree that data from our Remission Clinic $^{8}$ were contradicted by the results of the ORIENT (NCT00141453) ALTITUDE, ONTARGET (NCT00153101) and VANEPHRON-D (NCT00555217) trials. ORIENT and ALTITUDE tested olmesartan and aliskiren: drugs that the FDA has contraindicated in patients with diabetes because of their specific adverse cardiovascular effects. ${ }^{4,5,10}$ Data from ONTARGET are not pertinent as they apply to patients without proteinuric nephropathies. On the contrary, data from VA-NEPHRON-D confirm the finding from our Remission Clinic that dual RAS blockade may be less effective in patients with type 2 diabetes, overt proteinuria and renal insufficiency than in patients with nondiabetic proteinuric nephropathies. ${ }^{8}$ The "ever increasing number of patients who present with ESRD [end-stage renal disease]," together with evidence that the age of new incident patients on dialysis is progressively increasing confirms that more effective cardioprotection enables an increasing number of patients to survive long enough to progress to ESRD. In fact stabilization of the incidence of kidney failure over the last decade can be attributed to more efficient use of RAS inhibitors. ${ }^{11}$

Makani et al.'s point that spironolactone increases the risk of hyperkalaemia is not pertinent as their meta-analysis applies to ACE inhibitors, angiotensin-receptor blockers and renin inhibitors, but not to aldosterone antagonists. As for their statement that reduction in proteinuria "does not unequivocally lead to renal protection," the authors should consider that, independent of the considered therapy, trials in patients with proteinuric nephropathy have consistently shown that slower long-term progression of renal disease is invariably associated with a short-term reduction in proteinuria ${ }^{12,13}$ and lower residual levels of proteinuria, ${ }^{14}$ but never with worsening of proteinuria. ${ }^{15,16}$

Finally, data from our Remission Clinic show that, over 7-years of follow-up, two of $56(3.6 \%)$ patients on dual RAS blockade progressed to ESRD, compared with 17 of $56(30.4 \%)$ matched reference patients on ACE inhibitor monotherapy (Figure 1a). ${ }^{8}$ Another study showed stable glomerular filtration rate in patients on dual RAS blockade and worsening renal function in those on ACE inhibitor monotherapy (Figure 1b). ${ }^{9}$ In both studies dual RAS blockade was safe and 


\section{CORRESPONDENCE}
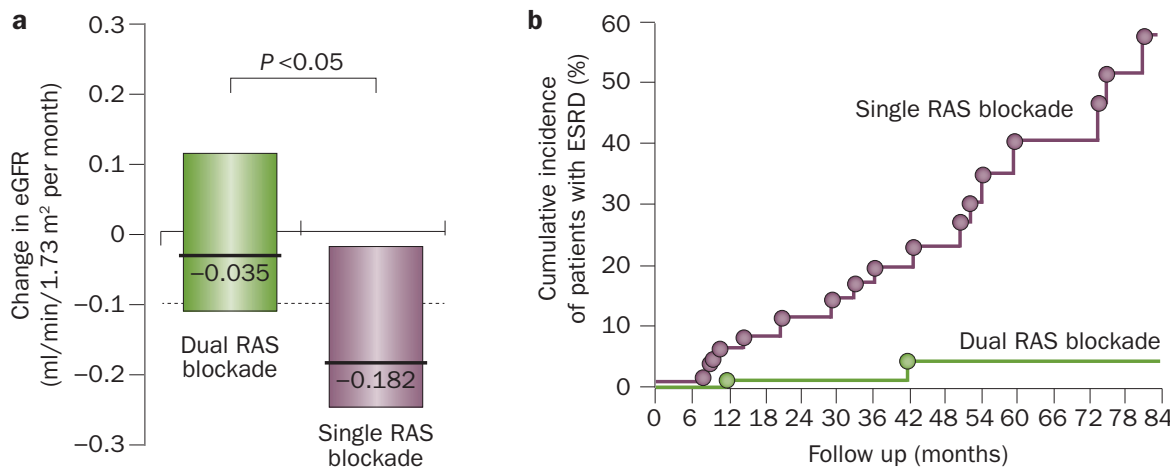

Figure 1 | Efficacy of dual (ACE inhibitor and ARB) versus single RAS blockade in patients with nephropathy. Reference patients were matched by age, gender and baseline proteinuria. a Rate of decline in eGFR over 6-year follow-up in 20 patients with nondiabetic proteinuric nephropathy on dual RAS blockade and in 20 reference patients on ACE inhibitor monotherapy. ${ }^{9}$ Data shown are the median and interquartile range. The dashed line indicates the physiological decline in eGFR in healthy individuals aged $>40$ years. b | Cumulative incidence of ESRD over 7-year follow-up in 56 patients with proteinuric chronic nephropathy on dual RAS blockade (Remission Clinic protocol) and in 56 reference patients on ACE inhibitor monotherapy. ${ }^{8}$ The excess risk of ESRD events in reference patients was significant (HR 0.092, 95\% Cl $0.021-0.401, P=0.0015)$ even after adjustment for gender, age, baseline serum creatinine and proteinuria (adjusted HR 0.027, 95\% Cl 0.004-0.173, $P=0.0001$ ). Circles identify ESRD events. Abbreviations: ACE, angiotensin-converting enzyme; ARB, angiotensin-receptor blocker; eGFR, estimated glomerular filtration rate; ESRD, end-stage renal disease; RAS, reninangiotensin system. Permission for part a obtained from Wichtig Editore $\odot$ Remission Clinic Task Force. The Remission Clinic approach to halt the progression of kidney disease. J. Nephrol. 24, 274-281 (2011). Permission for part b obtained from the American Society of Nephrology ( ) Ruggenenti, P. et al. Role of remission clinics in the longitudinal treatment of CKD. J. Am. Soc. Nephrol. 19, 1213-1224 (2008). Permissions conveyed through Copyright Clearance Center, Inc.

well tolerated. Thus, in patients with nondiabetic proteinuric nephropathies, evidence that the beneficial effects of dual RAS blockade on hard renal outcomes outstrip the well documented risk of adverse events is already available. Randomized controlled trials are, therefore, not required to further address this issue in this context. If they do not trust the available data, Makani and coworkers are ethically committed to personally conduct such a trial to protect patients from unnecessary exposure to potentially harmful treatments. In this perspective, it is intriguing to know whether, in the place of their patients, Makani et al. would consent to be randomly assigned to the control arm.
IRCCS-Istituto di Ricerche Farmacologiche Mario Negri, Centro Anna Maria Astori, Science and Technology Park Kilometro Rosso, Via Stezzano 87, 24126 Bergamo, Italy (P. Ruggenenti, G. Remuzzi).

Correspondence to: G. Remuzzi

giuseppe.remuzzi@marionegri.it

Competing interests

The authors declare no competing interests.

1. Ruggenenti, P. \& Remuzzi, G. Renin-angiotensin system: meta-analyses can misdirect decisions on treatment. Nat. Rev. Nephrol. 9, 311-312 (2013).

2. Makani, H., Bangalore, S., Desouza, K. A. Shah, A. \& Messerli, F. H. Efficacy and safety of dual blockade of the renin-angiotensin system: meta-analysis of randomised trials. BMJ 346, f360 (2013).
3. Makani, H. et al._Dual RAS blockadeunresolved controversy. Nat. Rev. Nephrol. http://dx.doi.org/10.1038/nrneph.2013.82-c1.

4. Parving, H. H. et al. Cardiorenal end points in a trial of aliskiren for type 2 diabetes. N. Engl. J. Med. 367, 2204-2213 (2012).

5. US Department of Health and Human Services. Aliskiren-containing medications: drug safety comunication-new warning and contraindication. US Food and Drug Administration [online], http://www.fda.gov/ Safety/MedWatch/Safetylnformation/ SafetyAlertsforHumanMedicalProducts/ ucm301120.htm (2012).

6. Noris, M. \& Remuzzi, G. Thrombotic microangiopathy: what not to learn from a metaanalysis. Nat. Rev. Nephrol. 5, 186-188 (2009).

7. Gay, J. Clinical epidemiology \& evidence-based medicine glossary: clinical study design and methods terminology. Washington State University College of Veterinary Medicine [online], http://www.vetmed.wsu.edu/ courses-jmgay/GlossClinStudy.htm (2010).

8. Ruggenenti, P. et al. Role of remission clinics in the longitudinal treatment of CKD. J. Am. Soc. Nephrol. 19, 1213-1224 (2008).

9. Remission Clinic Task Force. The Remission Clinic approach to halt the progression of kidney disease. J. Nephrol. 24, 274-281 (2011).

10. US Department of Health and Human Services. FDA Drug Safety Communication: safety review update of benicar (olmesartan) and cardiovascular events. US Food and Drug Administration [online], http://www.fda.gov/ Drugs/DrugSafety/ucm251268.htm (2011).

11. Ruggenenti, P. \& Remuzzi, G. Kidney failure stabilizes after a two-decade increase: impact on global (renal and cardiovascular) health. Clin. J. Am. Soc. Nephrol. 2, 146-150 (2007).

12. Ruggenenti, P. et al. Renal function and requirement for dialysis in chronic nephropathy patients on long-term ramipril: REIN follow-up trial. Gruppo Italiano di Studi Epidemiologici in Nefrologia (GISEN). Ramipril Efficacy in Nephropathy. Lancet 352, 1252-1256 (1998).

13. de Zeeuw, D. et al. Proteinuria, a target for renoprotection in patients with type 2 diabetic nephropathy: lessons from RENAAL. Kidney Int. 65, 2309-2320 (2004).

14. Ruggenenti, P., Perna, A. \& Remuzzi, G. Retarding progression of chronic renal disease: the neglected issue of residual proteinuria. Kidney Int. 63, 2254-2261 (2003).

15. Ruggenenti, P., Schieppati, A. \& Remuzzi, G Progression, remission, regression of chronic renal diseases. Lancet 357, 1601-1608 (2001).

16. Cravedi, P., Ruggenenti, P. \& Remuzzi, G. Proteinuria should be used as a surrogate in CKD. Nat. Rev. Nephrol. 8, 301-306 (2012). 